\title{
Research and Development of the Android Framework Smart Watches from the Data Security Point of View
}

\author{
Xin Xin Xie ${ }^{\mathrm{a}}$, Wen Zhun Huang ${ }^{\mathrm{b}^{*}}$ \\ ${ }^{1}$ Department of Electronic Information Engineering, Xijing University, Xi'an, 710123, China \\ aemail: 346148500@qq.com, bemail: huangwenzhun@xijing.edu.cn
}

Keywords: Android, Smart Watches, Data Security, Point of View, General Framework.

\begin{abstract}
In this paper, we conduct research on the Android framework smart watches from the data security point of view. With the speeding development of smart watches, Android smart watches the operating system is more and more attention by people and use, data security has become the user's concern problem, this article through to the Android system expatiates on that the various security mechanisms, and for different security mechanisms to provide the solution and hope to provide a little help developers improve Android system security. The scheme improves the Android data security, realizes the Android data encryption and authentication, ensures the confidentiality and integrity of the sensitive data, as well as resistance to deny as to the further popularization of the Android system provides a safety basis that is meaningful.
\end{abstract}

\section{Introduction}

In the early days of software architecture research, people on the system elements and their interaction gave high attention, but always ignore the interface elements like the interface is not part of the frame. Interface completely belong to the category of architecture, however, no interfaces, people cannot be analyzed system or the system building. Therefore, the software architecture view is a key on filing work for the view shown in the elements of the interface file. The interaction of elements and the basic environment can use a variety of the forms, most interactions involving control and data transmission, which requires the architect design interface and the specification to describe interfaces.

Based on the literature review we summarize the principles of software architecture and framework as the following aspects. (1) For software architecture, there is no a unified definition. It is defined as typical architecture of the program or computing system is the system of one or more of the structure, including software component, the component of external visible nature and the relationship between them. Architecture depicts the blueprint of system as it is communication software requirements and the software design of the bridge. (2) Software component technology is the core of software reuse. Component is in the system can clearly identify composition and the software component is software system has a certain significance of relatively independent composition; Reusable component refers to the independent function, on the basis of realizing the function of reusable components. (3) In the field of system analysis, including identification, collection, organization, analysis and presentation of related information, the commonness and individuality of form field definitions and the domain model and the domain model to determine the function of the system in the field of public and data according to the domain model, designed for specific areas of software architecture DSSA [1-4].

Smart watches, smart glasses for the advent of wearable devices have brought the new direction of the development, the Android system is widely used in intelligent wearable devices, smart phones. In order to make the wearable devices and mobile phones better cooperate with each other, meet people's needs. However, this does not meet the needs of users that users are more expectation through blue-tooth to realize the remote control function and the current blue-tooth data did not consider the content, is only simple data transfer, if we want to achieve blue-tooth remote control, you need to send a custom data structure. Control information and normal data information are 
transmitted between the devices via blue-tooth control information and data information in the existing added head node sends data first. Cloud security is the most prominent problems in data security, although each cloud service providers are stated, its services in all aspects that very safe. But to the enterprise, especially in large companies, and relevant data is the lifeblood of the business, cannot be threatened by any form. Shown in Figure1, we demonstrate the contemporary smart watches types.

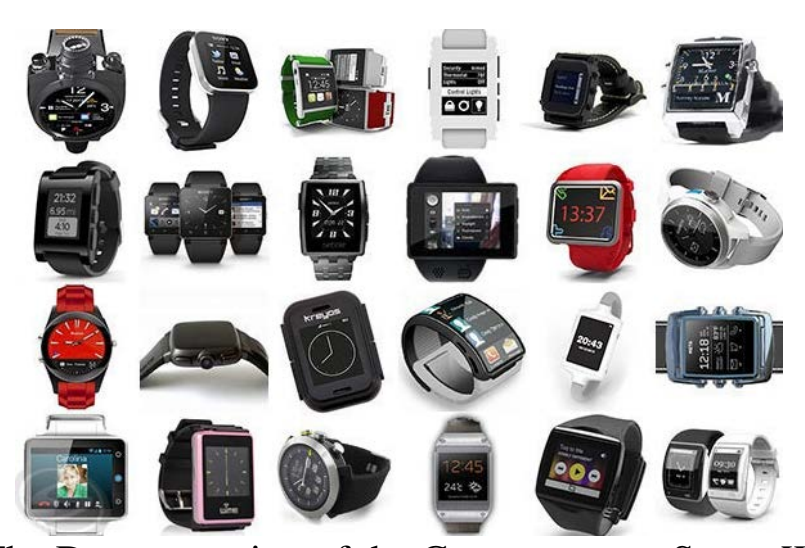

Fig.1. The Demonstration of the Contemporary Smart Watches

In this paper, we conduct research on the Android framework smart watches from the data security point of view. In the next sub-sections, we will discuss the issues in detail.

\section{Our Proposed Methodology}

The Software Architecture. All the parts of the software architecture describes the system software and their association with each other, it is neither the demand of the software application, also is not the concrete realization of software system, but the internal structure of software system configuration of an abstract description and it defines the interface specification of each part of the software system application and operation and data communications protocol and restrict each other.

Component structure is the inheritance of object-oriented structure and extension that it has all the advantages of the general object-oriented structure. At the same time, in this model, component is an encapsulated good feature that the foreign with consistent interface, the communication between the components by the bus unified agent, reduce the interdependence between the components. Software developers can easily define components and build a new plug and play to extend the system. The increase of the application components is easier, even during the system running dynamic loading and unloading. Application software package and enabled to allow system application software modules of software bus, and applications of all types of users start automatically as required.

- Component specifications including interface part and part structure, service of the interface set defines the component functions and the services provided by foreign. Port specification defines the characteristics of the dynamic behavior of components that the components and the external environment interaction information.

- A software system is composed of components and connectors as architecture configuration by the connector specification defines the components and the constraints of the basic external environment interaction [5-6].

- The structure of the atom component part defines the component implementation references. The structure of composite component part to define the relationship between the members of the connection between the components, including members of reference component types, members of component instance, connection between relationship and composite component interface specification to members of the component interface specification mapping.

The Data Security. From the point of service scope, cloud computing is divided into public cloud, private and the hybrid cloud. Public cloud services are usually all over the Internet, and to serve the almost unlimited number of general customers, provide a rich and colorful IT services and business 
applications. Private cloud are special customized for individual institutions that using the virtualized operating systems and network technology, to reduce the use of server and network equipment or the equipment management clearer that demonstrated in the Figure 2.

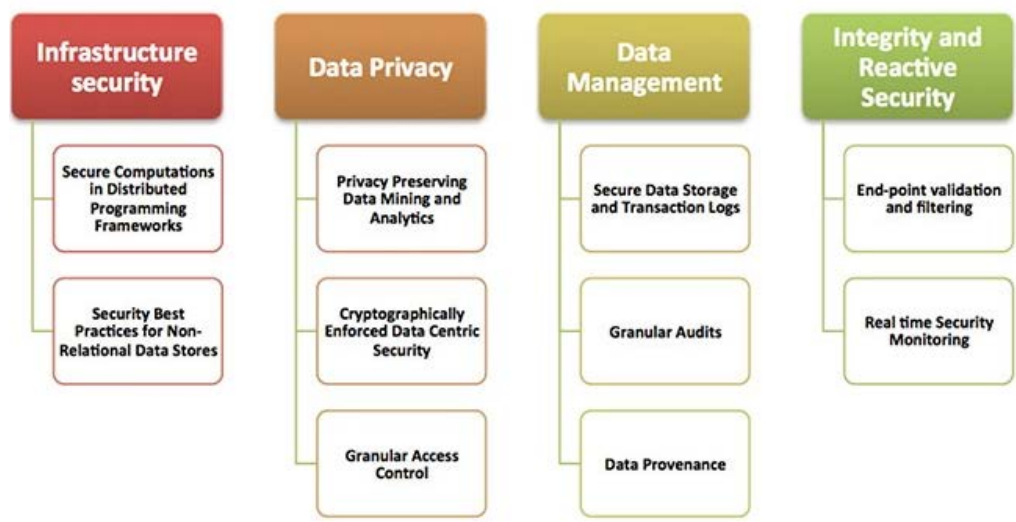

Fig.2. The Data Security Architecture and the Frameworks

Quality, energy and information are three basic attributes, is widespread in the objective world and man's conscious activity. The so-called information refers to the way things are or motion state, and this way or motion directly or indirectly. Information has timeliness, send feature, sharing as can be measured and controlled while we should follow the listed suggestions.

- Disaster information system strategy. It includes local disaster, basic disaster in another place, system management and the system recovery strategy. Disaster is the main means of the fault tolerance, the use of additional resources redundancy technology to achieve the effect of the masking the fault, which automatically recovery system or the harm to a minimum.

- System backup strategy. In most of the information system, the data distribution, large amount of data and backup server is more, the combination of the incremental and full back-up to the backup strategy to suit the general environment.

- Data encryption strategies. The effective information encryption measures that can make the attacker cannot understand, modify, sensitive information, even if important information intercepted, not leak, or the delay the time of disclosure. The user can according to the network situation, reasonably select the encryption method.

- Isolation strategy. Bad network isolation technology goal is to ensure that the isolation of core network attacks, guarantee the network under the premise of internal information is leaked, to complete the security exchange of data between networks [7].

Network security through related security technology to carry out practical protection, need through the establishment of a series of safety management system to do management work, in particular, we need to first do the good job in big data specification of construction for large data itself is an orderly and dynamic systematic engineering, its safe operation is the canonical operation mechanism for the escort as we also need to establish a data-centric security system that make big data in a safety system management structure, and through the wisdom innovation to the integration of big data and the cloud computing concepts, such as to building the large data fusion technology platform, thus better defense network security problems to ensure that under the large data of network safe and stable operation.

The Android Architecture and Safety. Android is a multitasking system, and each application or system program only in the process of its operation. To a certain extent, because the Android uses the Linux kernel, each application will be assigned user and group ID number, in this case, between the system and application security is greatly strengthened and Android like modify system files, such as special operations carried on the limits, only those who receive the user allows applications to be able to and the Android framework layer, Google has binding several security mechanisms.

The advantages of Android operating system is the system of each application in a corresponding virtual machine run independently, each other, there is no one program can access another program 
resources. Based on the Android version, the Android operating system does not provide built-in or the default encryption, user data protection relies mainly on the access patterns and isolated way to implement as this way without encryption protection makes the Android mobile phone once escaped from prison, so most of the mobile phone disclosure or loss of data.

- Android application framework layer provides limited access between general components of mandatory access control mechanism, a series of safety operation related permissions defined in system, applications need to declare themselves in the configuration file to use these labels required permissions, after the user agrees to authorize, the application of all component will inherit the application statement all the permissions. At the same time, the component can also use permissions can limit scope of components and their interaction.

- Linux discretionary access control mechanism to ensure that the application file is not visible to other applications. In particular, the Android application running in general Dalvik virtual machine, so with other application code is isolated.

- The activities of the Android framework layer manager is responsible for basic managing the application lifecycle, it contains a reference to the monitor if the caller is responsible for the validation of communication between components required by the caller. Package installer is responsible for the installation program, while a package manager is responsible for current maintaining all the application information in the system.

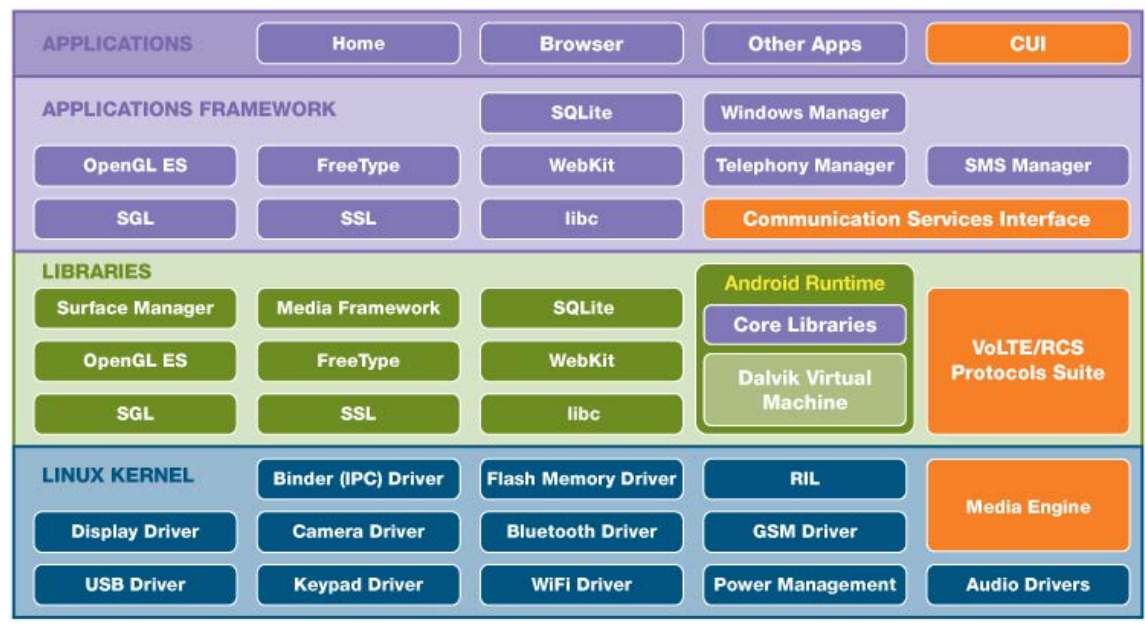

Fig.3. The Characteristics of the Android Framework

The Android Framework Smart Watches. Recently, domestic electricity Jingdong introduced its smart watches, and Nissan also promote will launch can connect the smart watch. It's at the same time, apple is expected to launch its first iWatch, and Google are also quietly to carry out her plan, acer, Intel, Microsoft, LG, etc. are all in the making. The personage inside course of the study bold predictions, may become the smart watch television screens, computer screens, mobile phone screen after the "fourth screen", lead the next wave of agitation of mobile Internet.

Although the mobile phone has increasingly tend to be light, but the user demand for large screen and high performance makes the volume of the mobile phone has not be able to significantly reduce room for the manoeuvre. In this case, if the part can be used in the mobile phone function separation according to wear with other electronic devices [8].

Meta Watch by Texas instruments MSP430TM processor and CC2560 blue-tooth chip, CC2560 ultra-low power consumption makes the Watch battery life guarantee that is understood to be a charge can maintain the use time of a week. In order to adapt to the needs of different users, the watch two different paragraphs using the pointer and digital watches is designed, which come with two pieces of white OLED screen, electronic watch is reflective screen, built-in blue-tooth, vibration, acceleration sensor, ambient light sensor. Especially worthy of the mark, the watch also has its own development platform, developers can through this platform further expansion of the mobile phone and watch the interaction between applications to meet different needs. For this issue, we recommend the following state-of-the-art smart watches as the guidelines for further development. 
- SONY Smartwatch2. Use 220 x 2201.6 inch screen, its waterproof level reached IP57. SONY said the watch of standby time than any competing goods on the market.

- AGENT. Agent used the 1.28 inch screen that smart watch glass covered the anti-glare. For the ARM processor architecture M4, low power consumption, support Qi wireless charging, movement and light sensors. In addition, it also can be waterproof, watch of wrist strap can be replaced, other functions include mobile phone left to surface can be downloaded to replace.

- WIMM One. This display screen has two kinds of operation mode, conventional color screen and reflective backlit screen. Under the condition of no backlighting can save electricity. It is also very convenient to carry and can be used to form a complete set of strap worn on hand as that also has a Micro USB interface of the base can connect the computer.

\section{Summary and Conclusion}

In this paper, we conduct research on the Android framework smart watches from the data security point of view. Although in comparison with the regulation of the app store, the Android Market app store regulatory scrutiny has yet to be improved, but its security is absolutely higher than ask file downloaded in the outside, and often within the store on Google some malware were forced off the shelves can also be downloaded from some special brand mobile phone app store procedures, the software in the market are usually tested, the compatibility and security is guaranteed. Under this core circumstances, we propose the novel Android framework smart watches from the data security point of view to optimize the robustness that will be meaningful.

\section{References}

[1] Bieber, Gerald, Marian Haescher, and Matthias Vahl. Sensor requirements for activity recognition on smart watches. Proceedings of the 6th International Conference on PErvasive Technologies Related to Assistive Environments. ACM, 2013.

[2] Esteves, Augusto, et al. Orbits: enabling gaze interaction in smart watches using moving targets. Proceedings of the 2015 ACM International Joint Conference on Pervasive and Ubiquitous Computing and Proceedings of the 2015 ACM International Symposium on Wearable Computers. ACM, 2015.

[3] Pirkl, Gerald, et al. mBeacon: accurate, robust proximity detection with smart phones and smart watches using low frequency modulated magnetic fields. Proceedings of the 10th EAI International Conference on Body Area Networks. ICST (Institute for Computer Sciences, Social-Informatics and Telecommunications Engineering), 2015.

[4] Hoelzl, Gerold, et al. Goal oriented smart watches for cyber physical superorganisms. Proceedings of the 2014 ACM International Joint Conference on Pervasive and Ubiquitous Computing: Adjunct Publication. ACM, 2014.

[5] Poirier, Franck, and Mohammed Belatar. UniWatch-Some Approaches Derived from UniGlyph to Allow Text Input on Tiny Devices Such as Connected Watches. Human-Computer Interaction: Interaction Technologies. Springer International Publishing, 2015. 554-562.

[6] Xie, Mimi, et al. Fixing the broken time machine: consistency-aware checkpointing for energy harvesting powered non-volatile processor. Proceedings of the 52nd Annual Design Automation Conference. ACM, 2015.

[7] Alameda-Pineda, Xavier, et al. Analyzing free-standing conversational groups: a multimodal approach. Proceedings of the 23rd Annual ACM Conference on Multimedia Conference. ACM, 2015. 
[8] Wang, Hao, et al. A 5.4-mW 180-cm transmission distance 2.5-Mb/s advanced techniques-based novel intrabody communication receiver analog front end. Very Large Scale Integration (VLSI) Systems, IEEE Transactions on 23.12 (2015): 2829-2841. 